\title{
Certain Subclasses of Analytic Functions with Complex Order
}

\author{
A. Selvam, ${ }^{1}$ P. Sooriya Kala, ${ }^{1}$ and N. Marikkannan ${ }^{2}$ \\ ${ }^{1}$ Department of Mathematics, VHNSN College, Virudhunagar 626001, India \\ ${ }^{2}$ Department of Mathematics, Government Arts College, Melur 625106, India
}

Correspondence should be addressed to N. Marikkannan; natarajan.marikkannan@gmail.com

Received 12 August 2013; Accepted 12 September 2013

Academic Editors: G. Bonanno and A. Ibeas

Copyright (c) 2013 A. Selvam et al. This is an open access article distributed under the Creative Commons Attribution License, which permits unrestricted use, distribution, and reproduction in any medium, provided the original work is properly cited.

Two new subclasses of analytic functions of complex order are introduced. Apart from establishing coefficient bounds for these classes, we establish inclusion relationships involving $(n-\delta)$ neighborhoods of analytic functions with negative coefficients belonging to these subclasses.

\section{Introduction}

Let $\mathscr{A}(n)$ denote the class of functions of the form

$$
f(z)=z-\sum_{k=n+1}^{\infty} a_{k} z^{k} \quad\left(a_{k} \geq 0, n \in \mathbb{N}:=\{1,2,3, \ldots\}\right),
$$

which are analytic and univalent in the open disc

$$
\Delta=\{z \in \mathbb{C}:|z|<1\} .
$$

A function $f(z) \in \mathscr{A}(n)$ is star-like of complex order $b$, denoted as $f(z) \in S^{*}(b)$ if and only if it satisfies

$$
\mathfrak{R}\left\{1+\frac{1}{b}\left(\frac{z f^{\prime}}{f}-1\right)\right\}>0 \quad(z \in \Delta)
$$

A function $f(z) \in \mathscr{A}(n)$ is convex of complex order $b$, denoted as $f(z) \in C(b)$ if and only if it satisfies

$$
\Re\left\{1+\frac{1}{b}\left(\frac{z f^{\prime \prime}}{f^{\prime}}\right)\right\}>0 \quad(z \in \Delta) .
$$

These classes, $S^{*}(b)$, and $C(b)$ are introduced and studied by Nasr and Aouf [1] and Wiatrowski [2].

For the two functions $f_{j}(j=1,2)$ given by

$$
f_{j}(z)=z+\sum_{k=2}^{\infty} a_{k, j} z^{k} \quad(j=1,2)
$$

the Hadamard product or convolution, denoted by $\left(f_{1} *\right.$ $\left.f_{2}\right)(z)$, is given by

$$
\left(f_{1} * f_{2}\right)(z)=z+\sum_{k=2}^{\infty} a_{k, 1} a_{k, 2} z^{k}
$$

Given $f(z)$ of the form (1) and $\delta \geq 0$, we define $n-\delta$ neighborhood of a function $f \in \mathscr{A}(n)$ as

$$
N_{n, \delta}(f)=\left\{g \in \mathscr{A}(n)\left|\sum_{k=n+1}^{\infty} k\right| a_{k}-b_{k} \mid \leq \delta\right\} .
$$

In particular, for the identity function $e(z)=z$,

$$
N_{n, \delta}(e)=\left\{g \in \mathscr{A}(n)\left|\sum_{k=n+1}^{\infty} k\right| b_{k} \mid \leq \delta\right\} .
$$

The concept of Neighborhood $N_{n, \delta}$ of a function $f$ is introduced and studied by Ruscheweyh [3] and extended further by Silverman [4].

For complex numbers $\alpha_{1}, \alpha_{2}, \ldots, \alpha_{q}$ and $\beta_{1}, \beta_{2}, \ldots, \beta_{s}$ $\left(\beta_{j} \in \mathbb{C} \backslash \mathscr{Z}_{0}^{-} ; \mathscr{Z}_{0}^{-}=\{0,-1,-2, \ldots\}\right.$ for $\left.j=1,2, \ldots, s\right)$, 
we define the generalized hypergeometric function ${ }_{q} F_{s}\left(\alpha_{1}, \alpha_{2}, \ldots, \alpha_{q} ; \beta_{1}, \beta_{2}, \ldots, \beta_{s} ; z\right)$ as

$$
\begin{aligned}
& { }_{q} F_{s}\left(\alpha_{1}, \alpha_{2}, \ldots, \alpha_{q} ; \beta_{1}, \beta_{2}, \ldots, \beta_{s} ; z\right) \\
& =\sum_{k=0}^{\infty} \frac{\left(\alpha_{1}\right)_{k}\left(\alpha_{2}\right)_{k} \cdots\left(\alpha_{q}\right)_{k} z^{k}}{\left(\beta_{1}\right)_{k}\left(\beta_{2}\right)_{k} \cdots\left(\beta_{s}\right)_{k} k !}, \quad(q \leq s+1 ;
\end{aligned}
$$$$
\left.q, s \in \mathbb{N}_{0}:=\mathbb{N} \cup\{0\} ; z \in U\right) \text {, }
$$

where $\mathbb{N}$ denotes the set of all positive integers and $(x)_{k}$ is the Pochhammer symbol defined in terms of gamma functions as

$$
(x)_{k}=\frac{\Gamma(x+k)}{\Gamma(x)}= \begin{cases}1 & \text { if } k=0 \\ x(x+1) \cdots(x+k-1) & \text { if } k \in \mathbb{N}\end{cases}
$$

Corresponding to the function $g_{q, s}\left(\alpha_{1}, \beta_{1} ; z\right)$ defined by

$$
\begin{aligned}
g_{q, s} & \left(\alpha_{1}, \beta_{1} ; z\right) \\
& =z_{q} F_{s}\left(\alpha_{1}, \alpha_{2}, \ldots, \alpha_{q} ; \beta_{1}, \beta_{2}, \ldots, \beta_{s} ; z\right)
\end{aligned}
$$

recently in [5], an operator $\mathscr{D}_{\lambda, \mu}^{m}\left(\alpha_{1}, \beta_{1}\right) f(z): \mathscr{A} \rightarrow \mathscr{A}$ is defined by

$$
\begin{aligned}
& \mathscr{D}_{\lambda, \mu}^{0}\left(\alpha_{1}, \beta_{1}\right) f(z):=f(z) * g_{q, s}\left(\alpha_{1}, \beta_{1} ; z\right) \\
& \mathscr{D}_{\lambda, \mu}^{1}\left(\alpha_{1}, \beta_{1}\right) f(z):=(1-\lambda+\mu)\left(f(z) * g_{q, s}\left(\alpha_{1}, \beta_{1} ; z\right)\right) \\
&+(\lambda-\mu) z\left(f(z) * g_{q, s}\left(\alpha_{1}, \beta_{1} ; z\right)\right)^{\prime} \\
&+\lambda \mu z^{2}\left(f(z) * g_{q, s}\left(\alpha_{1}, \beta_{1} ; z\right)\right)^{\prime \prime} \\
& \mathscr{D}_{\lambda, \mu}^{m}\left(\alpha_{1}, \beta_{1}\right) f(z):=\mathscr{D}_{\lambda, \mu}^{1}\left(\mathscr{D}_{\lambda, \mu}^{m-1}\left(\alpha_{1}, \beta_{1}\right) f(z)\right)
\end{aligned}
$$

where $0 \leq \mu \leq \lambda \leq 1$ and $m \in \mathbb{N}_{0}$. By the above definition, it is easy to note that

$$
\begin{aligned}
\mathscr{D}_{\lambda, \mu}^{m}\left(\alpha_{1}, \beta_{1}\right) f(z) \\
=z+\sum_{k=2}^{\infty}[1+(k-1)(\lambda-\mu+k \mu \lambda)]^{m} \\
\quad \times \frac{\left(\alpha_{1}\right)_{k-1}\left(\alpha_{2}\right)_{k-1} \ldots\left(\alpha_{q}\right)_{k-1}}{\left(\beta_{1}\right)_{k-1}\left(\beta_{2}\right)_{k-1} \ldots\left(\beta_{s}\right)_{k-1}(k-1) !} a_{k} z^{k} .
\end{aligned}
$$

Let us take for convenience that

$$
\begin{gathered}
B_{k}=\frac{\left(\alpha_{1}\right)_{k-1}\left(\alpha_{2}\right)_{k-1} \ldots\left(\alpha_{q}\right)_{k-1}}{\left(\beta_{1}\right)_{k-1}\left(\beta_{2}\right)_{k-1} \ldots\left(\beta_{s}\right)_{k-1}(k-1) !}, \\
C_{k}=1+(k-1)(\lambda-\mu+k \mu \lambda) .
\end{gathered}
$$

Hence, we have

$$
\mathscr{D}_{\lambda, \mu}^{m}\left(\alpha_{1}, \beta_{1}\right) f(z)=z+\sum_{k=2}^{\infty} C_{k}^{m} B_{k} a_{k} z^{k}
$$

For suitable values of $\alpha_{i^{\prime} s}, \beta_{j^{\prime} s}, q, s, m, \lambda$, and $\mu$, we can deduce several operators such as Sălăgean derivative operator [6], Ruscheweyh derivative operator [7], fractional calculus operator [8], Carlson-Shaffer operator [9], Dziok-Srivatsava operator [10], and also the operator introduced by Abubaker and Darus [11].

Definition 1. For $0 \leq \alpha \leq 1$, we let $A$ be the subclass of $\mathscr{A}(n)$ consisting of functions of the form (1) that satisfy

$$
\begin{aligned}
& \mid \frac{1}{b}\left(z\left[\mathscr{D}_{\lambda, \mu}^{m}\left(\alpha_{1}, \beta_{1}\right) f(z)\right]^{\prime}\right. \\
& \quad \times\left((1-\alpha) \mathscr{D}_{\lambda, \mu}^{m}\left(\alpha_{1}, \beta_{1}\right) f(z)\right. \\
& \left.\left.\quad+\alpha z\left[\mathscr{D}_{\lambda, \mu}^{m}\left(\alpha_{1}, \beta_{1}\right) f(z)\right]^{\prime}\right)^{-1}\right) \mid<\gamma,
\end{aligned}
$$

where $z \in \Delta, b \in \mathbb{C} \backslash\{0\}, 0<\gamma \leq 1$, and $\mathscr{D}_{\lambda, \mu}^{m}\left(\alpha_{1}, \beta_{1}\right) f(z)$ are as given in (15).

Definition 2. For $0 \leq \alpha \leq 1$ we let $B$ be the subclass of $\mathscr{A}(n)$, consisting of functions of the form (1) that satisfy

$$
\begin{aligned}
& \quad \mid \frac{1}{b}\left((1-\alpha) \frac{\mathscr{D}_{\lambda, \mu}^{m}\left(\alpha_{1}, \beta_{1}\right) f(z)}{z}\right. \\
& \left.\quad+\alpha\left[\mathscr{D}_{\lambda, \mu}^{m}\left(\alpha_{1}, \beta_{1}\right) f(z)\right]^{\prime}-1\right) \mid<\gamma,
\end{aligned}
$$

where $z \in \Delta, b \in \mathbb{C} \backslash\{0\}, 0<\gamma \leq 1$, and $\mathscr{D}_{\lambda, \mu}^{m}\left(\alpha_{1}, \beta_{1}\right) f(z)$ are as given in (15).

By specializing the parameters involved in the above definitions, we could arrive at several known as well as new classes. For example, by taking $\lambda=1, \mu=0, q=2, s=1$, $\alpha_{1}=\beta_{1}$, and $\alpha_{2}=1$ and the above classes reduced to

$$
A_{1}=\left\{f \in \mathscr { A } ( n ) | | \frac { 1 } { b } \left(z\left[\mathbb{D}^{m} f(z)\right]^{\prime}\right.\right.
$$

$$
\begin{aligned}
& \times\left((1-\alpha) \mathbb{D}^{m} f(z)\right. \\
& \left.\left.\left.\quad+\alpha z\left[\mathbb{D}^{m} f(z)\right]^{\prime}\right)^{-1}\right) \mid<\gamma\right\},
\end{aligned}
$$


where $\mathbb{D}^{m} f(z)$ denote the Sălăgean derivative of order $m$ given by

$$
\begin{gathered}
\mathbb{D}^{m} f(z)=z-\sum_{k=n+1}^{\infty} k^{m} a_{k} z^{k}, \\
B_{1}=\left\{f \in \mathscr { A } ( n ) | | \frac { 1 } { b } \left((1-\alpha) \frac{\mathbb{D}^{m} f(z)}{z}\right.\right. \\
\left.\left.+\alpha\left[\mathscr{D}^{m} f(z)\right]^{\prime}-1\right) \mid<\gamma\right\} .
\end{gathered}
$$

Similarly, on taking $q=2, s=1, \alpha_{1}=\eta-1,(\eta>-1)$, $\alpha_{2}=1, \beta_{1}=1$, one gets

$$
\begin{aligned}
A_{2}=\left\{f \in \mathscr{A}(n)|| \frac{1}{b}(\right. & z\left[D^{m} f(z)\right]^{\prime} \\
\times & \left((1-\alpha) D^{m} f(z)\right. \\
& \left.\left.\left.+\alpha z\left[D^{m} f(z)\right]^{\prime}\right)^{-1}\right) \mid<\gamma\right\},
\end{aligned}
$$

where $D^{m} f(z)$ is the operator introduced and studied by Abubaker and Darus [11] given by

$$
\begin{gathered}
D^{m} f(z)=z-\sum_{k=n+1}^{\infty} C_{k}^{m}\left(\begin{array}{c}
\eta+k-1 \\
k-1
\end{array}\right) z^{k}, \\
B_{2}=\left\{f \in \mathscr { A } ( n ) | | \frac { 1 } { b } \left(\begin{array}{c}
(1-\alpha) \frac{D^{m} f(z)}{z} \\
\left.\left.+\alpha\left[D^{m} f(z)\right]^{\prime}-1\right) \mid<\gamma\right\} .
\end{array}\right.\right.
\end{gathered}
$$

Further, by taking $m=0$ in the definition of the classes $A$ and $B$, we could arrive at $S_{n}(q, s, \alpha, b, \gamma)$ and $R_{n}(q, s, \alpha, b, \gamma)$ which were introduced and studied by Murugusundaramoorthy et al. [12].

In this paper, we establish the coefficient inequalities for the classes $A$ and $B$ and several inclusion relationships involving $n-\delta$ neighborhoods of analytic univalent functions with negative and missing coefficients belonging to these classes.

\section{Coefficient Inequalities}

Theorem 3. Let the function $f \in \mathscr{A}(n)$ as given in (1). Then, $f \in A$ if and only if

$$
\sum_{k=n+1}^{\infty}\{[1+\alpha(k-1)](\gamma|b|-1)+k\} C_{k}^{m} B_{k} a_{k} \leq \gamma|b| .
$$

Proof. Let the functions of form (1) belong to the class $A$. Then, in view of (15) and (16), we get

$$
\begin{aligned}
& \left|\frac{\sum_{k=n+1}^{\infty}([1+\alpha(k-1)]-k) C_{k}^{m} B_{k} a_{k} z^{k}}{z-\sum_{k=n+1}^{\infty}[1+\alpha(k-1)] C_{k}^{m} B_{k} a_{k} z^{k}}\right|<\gamma|b|, \\
& \frac{\sum_{k=n+1}^{\infty}([1+\alpha(k-1)]-k) C_{k}^{m} B_{k} a_{k} r^{k-1}}{1-\sum_{k=n+1}^{\infty}[1+\alpha(k-1)] C_{k}^{m} B_{k} a_{k} r^{k-1}}<\gamma|b| .
\end{aligned}
$$

Letting $r \rightarrow 1^{-}$through real values, we get the required assertion (22). Conversely, suppose $f(z)$ satisfies (22), then, in view of (16), consider

$$
\begin{aligned}
& \mid z\left[\mathscr{D}_{\lambda, \mu}^{m}\left(\alpha_{1}, \beta_{1}\right) f(z)\right]^{\prime} \\
& -(1-\alpha) \mathscr{D}_{\lambda, \mu}^{m}\left(\alpha_{1}, \beta_{1}\right) f(z) \\
& -\alpha z\left[\mathscr{D}_{\lambda, \mu}^{m}\left(\alpha_{1}, \beta_{1}\right) f(z)\right]^{\prime} \mid \\
& -\gamma|b| \mid(1-\alpha) \mathscr{D}_{\lambda, \mu}^{m}\left(\alpha_{1}, \beta_{1}\right) f(z) \\
& \quad+\alpha z\left[\mathscr{D}_{\lambda, \mu}^{m}\left(\alpha_{1}, \beta_{1}\right) f(z)\right]^{\prime} \mid \\
& =\left|\sum_{k=n+1}^{\infty}([1+\alpha(k-1)]-k) C_{k}^{m} B_{k} a_{k} z^{k}\right| \\
& \quad-\gamma|b|\left|z-\sum_{k=n+1}^{\infty}[1+\alpha(k-1)] C_{k}^{m} B_{k} a_{k} z^{k}\right| \\
& <\sum_{k=n+1}^{\infty}\{([1+\alpha(k-1)](\gamma|b|-1)+k)\} \\
& \leq 0 . \quad \times C_{k}^{m} B_{k} a_{k}-\gamma|b|
\end{aligned}
$$

Hence, the result follows.

Similarly, we prove the following.

Theorem 4. Let the function $f \in \mathscr{A}(n)$ be as defined in (1). Then, $f \in B$ if and only if

$$
\sum_{k=n+1}^{\infty}[1+\alpha(k-1)] C_{k}^{m} B_{k} a_{k} \leq \gamma|b| .
$$

Corollary 5. Let the function $f \in \mathscr{A}(n)$ as given in (1). Then, $f(z) \in A_{1}$ if and only if

$$
\sum_{k=n+1}^{\infty}\{[1+\alpha(k-1)](\gamma|b|-1)+k\} k^{m} a_{k} \leq \gamma|b| .
$$

Corollary 6. Let the function $f \in \mathscr{A}(n)$ be as defined in (1). Then, $f \in B_{1}$ if and only if

$$
\sum_{k=n+1}^{\infty}[1+\alpha(k-1)] k^{m} a_{k} \leq \gamma|b| .
$$

Corollary 7. Let the function $f \in \mathscr{A}(n)$ as given in (1). Then, $f \in A_{2}$ if and only if

$$
\begin{gathered}
\sum_{k=n+1}^{\infty}\{[1+\alpha(k-1)](\gamma|b|-1)+k\} C_{k}^{m} \\
\times\left(\begin{array}{c}
\eta+k-1 \\
k-1
\end{array}\right) a_{k} \leq \gamma|b| .
\end{gathered}
$$


Corollary 8. Let the function $f(z) \in \mathscr{A}(n)$ be as defined in (1). Then, $f \in B_{2}$ if and only if

$$
\sum_{k=n+1}^{\infty}[1+\alpha(k-1)] C_{k}^{m}\left(\begin{array}{c}
\eta+k-1 \\
k-1
\end{array}\right) a_{k} \leq \gamma|b| .
$$

\section{Inclusion Relationships}

Theorem 9. If

$$
\delta=\frac{\gamma|b|(1+n)}{[(1+n \alpha)(\gamma|b|-1)+n+1] B_{n+1} C_{n+1}^{m}} \quad(\gamma|b|>1),
$$

then $A \subset N_{n, \delta}(e)$.

Proof. Let $f \in A$. Then, in view of (22), we have

$$
\begin{gathered}
C_{n+1}^{m} B_{n+1}[(1+n \alpha)(\gamma|b|-1) n+1] \sum_{k=n+1}^{\infty} a_{k} \\
\leq \gamma|b|, \\
\sum_{k=n+1}^{\infty} a_{k} \leq \frac{\gamma|b|}{C_{n+1}^{m} B_{n+1}[(1+n \alpha)(\gamma|b|-1) n+1]} .
\end{gathered}
$$

Consider

$$
\begin{aligned}
C_{n+1}^{m} B_{n+1} & \sum_{k=n+1}^{\infty} k a_{k} \\
& \leq \gamma|b|+(1+n \alpha)(1-\gamma|b|) B_{n+1} C_{n+1}^{m} \sum_{k=n+1}^{\infty} a_{k} \\
& \leq \gamma|b|+\frac{(1+n \alpha)(1-\gamma|b|) \gamma|b|}{(1+n \alpha)(\gamma|b|-1)+n+1} \\
& \leq \frac{\gamma|b|(n+1)}{(1+n \alpha)(\gamma|b|-1)+n+1} .
\end{aligned}
$$

Hence,

$$
\begin{aligned}
\sum_{k=n+1}^{\infty} k a_{k} & \leq \frac{\gamma|b|(n+1)}{[(1+n \alpha)(\gamma|b|-1)+n+1] B_{n+1} C_{n+1}^{m}} \\
& =\delta .
\end{aligned}
$$

Hence, the result follows.

In similar manner, we establish the following result.

Theorem 10. If

$$
\delta=\frac{\gamma|b|(1+n)}{(1+n \alpha) B_{n+1} C_{n+1}^{m}},
$$

then $B \subset N_{n, \delta}(e)$.
Corollary 11. If

$$
\delta=\frac{\gamma|b|(1+n)}{[(1+n \alpha)(\gamma|b|-1)+n+1](n+1)^{m}},
$$

then $A_{1} \subset N_{n, \delta}(e)$.

Corollary 12. If

$$
\delta=\frac{\gamma|b|(1+n)}{(1+n \alpha)(n+1)^{m}},
$$

then $B_{1} \subset N_{n, \delta}(e)$.

Corollary 13. If

$$
\delta=\frac{\gamma|b|(1+n)}{[(1+n \alpha)(\gamma|b|-1)+n+1] C_{n+1}^{m}\left(\begin{array}{c}
n+n \\
n
\end{array}\right)},
$$

then $A_{2} \subset N_{n, \delta}(e)$.

Corollary 14. If

$$
\delta=\frac{\gamma|b|(1+n)}{(1+n \alpha) C_{n+1}^{m}\left(\begin{array}{c}
n+n \\
n
\end{array}\right)},
$$

then $B_{2} \subset N_{n, \delta}(e)$.

\section{Neighborhoods for $A^{\sigma}$ and $B^{\sigma}$}

In this section, we determine the neighborhood properties of $A^{\sigma}$ and $B^{\sigma}$. Here, the classes $A^{\sigma}$ consist of functions $f \in \mathscr{A}(n)$ for which there exists a function $g(z) \in A$ such that

$$
\left|\frac{f(z)}{g(z)}-1\right|<1-\sigma \quad(z \in \Delta, 0 \leq \sigma<1) .
$$

In the same way, we define $B^{\sigma}$, consisting of functions $f(z) \in$ $\mathscr{A}(n)$ for which there exists another function $g(z) \in B$ such that

$$
\left|\frac{f(z)}{g(z)}-1\right|<1-\sigma \quad(z \in \Delta, 0 \leq \sigma<1) .
$$

Theorem 15. If $g \in A$ and

$$
\begin{aligned}
\sigma=1- & \frac{\delta}{n+1} \\
\times & \{[(1+n \alpha)(\gamma|b|-1)+n+1] \\
& \times B_{n+1} C_{n+1}^{m} \\
& \times([(1+n \alpha)(\gamma|b|-1)+n+1] \\
& \left.\left.\times B_{n+1} C_{n+1}^{m}-\gamma|b|\right)^{-1}\right\} \\
& (\gamma|b|>1),
\end{aligned}
$$

then $N_{n, \delta}(g) \subset A^{\sigma}$. 
Proof. Suppose $f \in N_{n, \delta}(g)$, then

$$
\begin{gathered}
\sum_{k=n+1}^{\infty} k\left|a_{k}-b_{k}\right| \leq \delta, \\
\sum_{k=n+1}^{\infty}\left|a_{k}-b_{k}\right| \leq \frac{\delta}{n+1} .
\end{gathered}
$$

Since $g \in A$, we have

$$
\sum_{k=n+1}^{\infty} b_{k} \leq \frac{b|k|}{[(1+n \alpha)(\gamma|b|-1)+n+1] B_{n+1} C_{n+1}^{m}}
$$

Consider

$$
\begin{aligned}
& \left|\frac{f(z)}{g(z)}-1\right| \\
& \quad<\frac{\sum_{k=n+1}^{\infty}\left|a_{k}-b_{k}\right|}{1-\sum_{k=n+1}^{\infty} b_{k}} \\
& \quad \leq \frac{\delta}{n+1}\left\{\frac{[(1+n \alpha)(\gamma|b|-1)+n+1] B_{n+1} C_{n+1}^{m}}{[(1+n \alpha)(\gamma|b|-1)+n+1] B_{n+1} C_{n+1}^{m}-\gamma|b|}\right\} \\
& \quad=1-\sigma .
\end{aligned}
$$

Therefore, $f \in A^{\sigma}$ for $\sigma$ given by (42).

Theorem 16. If $g \in B$ and

$$
\sigma=1-\frac{\delta}{n+1}\left\{\frac{(1+n \alpha) B_{n+1} C_{n+1}^{m}}{(1+n \alpha) B_{n+1} C_{n+1}^{m}-\gamma|b|}\right\},
$$

then $N_{n, \delta}(g) \subset B^{\sigma}$.

Corollary 17. If $g \in A_{1}$ and

$$
\begin{gathered}
\sigma=1-\frac{\delta}{n+1}\left\{[(1+n \alpha)(\gamma|b|-1)+n+1](n+1)^{m}\right. \\
\times([(1+n \alpha)(\gamma|b|-1)+n+1] \\
\left.\left.\times(n+1)^{m}-\gamma|b|\right)^{-1}\right\} \\
(\gamma|b|>1),
\end{gathered}
$$

then $N_{n, \delta}(g) \subset A_{1}^{\sigma}$.

Corollary 18. If $g \in B_{1}$ and

$$
\sigma=1-\frac{\delta}{n+1}\left\{\frac{(1+n \alpha)(n+1)^{m}}{(1+n \alpha)(n+1)^{m}-\gamma|b|}\right\},
$$

then $N_{n, \delta}(g) \subset B_{1}^{\sigma}$.
Corollary 19. If $g \in A_{2}$ and

$$
\begin{gathered}
\sigma=1-\frac{\delta}{n+1}\left\{[(1+n \alpha)(\gamma|b|-1)+n+1]\left(\begin{array}{c}
\eta+n \\
n
\end{array}\right) C_{n+1}^{m}\right. \\
\times([(1+n \alpha)(\gamma|b|-1)+n+1] \\
\left.\left.\times\left(\begin{array}{c}
\eta+n \\
n
\end{array}\right) C_{n+1}^{m}-\gamma|b|\right)^{-1}\right\} \\
(\gamma|b|>1),
\end{gathered}
$$

then $N_{n, \delta}(g) \subset A_{2}^{\sigma}$.

Corollary 20. If $g \in B_{2}$ and

$$
\sigma=1-\frac{\delta}{n+1}\left\{\frac{(1+n \alpha)\left(\begin{array}{c}
\eta+n \\
n
\end{array}\right) C_{n+1}^{m}}{(1+n \alpha)\left(\begin{array}{c}
\eta+n \\
n
\end{array}\right) C_{n+1}^{m}-\gamma|b|}\right\},
$$

then $N_{n, \delta}(g) \subset B_{2}^{\sigma}$.

\section{Conflict of Interests}

The authors declare that they do not have conflict of interests regarding the publication of this paper.

\section{References}

[1] M. Nasr and M. Aouf, "Starlike functions of complex order," Journal of Natural Sciences and Mathematics, vol. 25, no. 1, pp. $1-12,1985$.

[2] P. Wiatrowski, "On the coefficients of some family of holomorphic functions," Zeszyty Naukowe, vol. 2, no. 39, pp. 75-85, 1970.

[3] St. Ruscheweyh, "Neighborhoods of univalent functions," Proceedings of the American Mathematical Society, pp. 521-527, 1981.

[4] H. Silverman, "Neighborhoods of a class of analytic functions," Far East Journal of Mathematical Sciences, vol. 3, no. 2, pp. 165169, 1995.

[5] N. Marikkannan, "A subclass of analytic functions anda generalised differential operator," communicated.

[6] G. S. Salagean, "Subclasses of univalent functions," in Complex Analysis, vol. 1013 of Lecture Notes in Mathematics, pp. 362372, Berlin, Germany, 1981, Proceedings of the 5th RomanianFinnish Seminar, Part 1, Bucharest, Romania, 1981.

[7] St. Ruscheweyh, "New criteria for univalent functions," Proceeding of the American Mathematical Society, vol. 49, pp. 109-115, 1975.

[8] S. Owa, "Some applications of the fractional calculus," in Proceedings of the Workshop on Fractional Calculus, vol. 138 of Research Notes in Mathematics, pp. 164-175, University of Strathclyde, Ross Priory, UK, 1985.

[9] B. C. Carlson and S. B. Shaffer, "Starlike and Prestarlike hypergeometric functions," SIAM Journal on Mathematical Analysis, vol. 15, no. 4, pp. 737-745, 2002.

[10] J. Dziok and H. M. Srivatsava, "Classes of analytic functions associated with the generalized hypergeometric function," Applied Mathematics and Computation, vol. 103, no. 1, pp. 1-13, 1999. 
[11] A. A. A. Abubaker and M. Darus, "Neighborhoods of certain classes of analytic functions defined by a generalized differential operator," International Journal of Mathematical Analysis, vol. 4, no. 45-48, pp. 2373-2380, 2010.

[12] G. Murugusundaramoorthy, T. Rosy, and S. Sivasubramanian, "On certain classes of analytic functions of complex order defined by Dziok-Srivatsava operator," Journal of Mathematical Inequalities, 2007. 


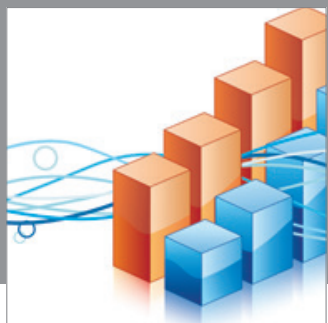

Advances in

Operations Research

mansans

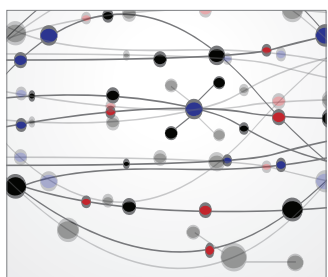

The Scientific World Journal
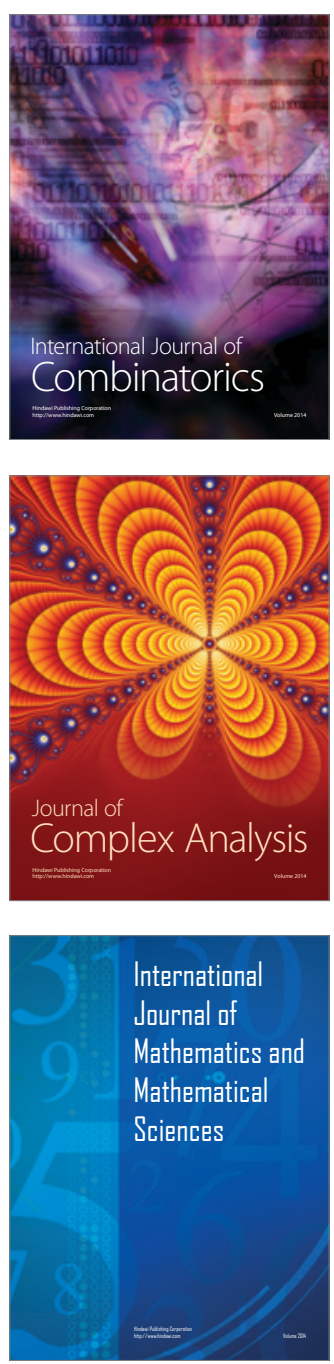
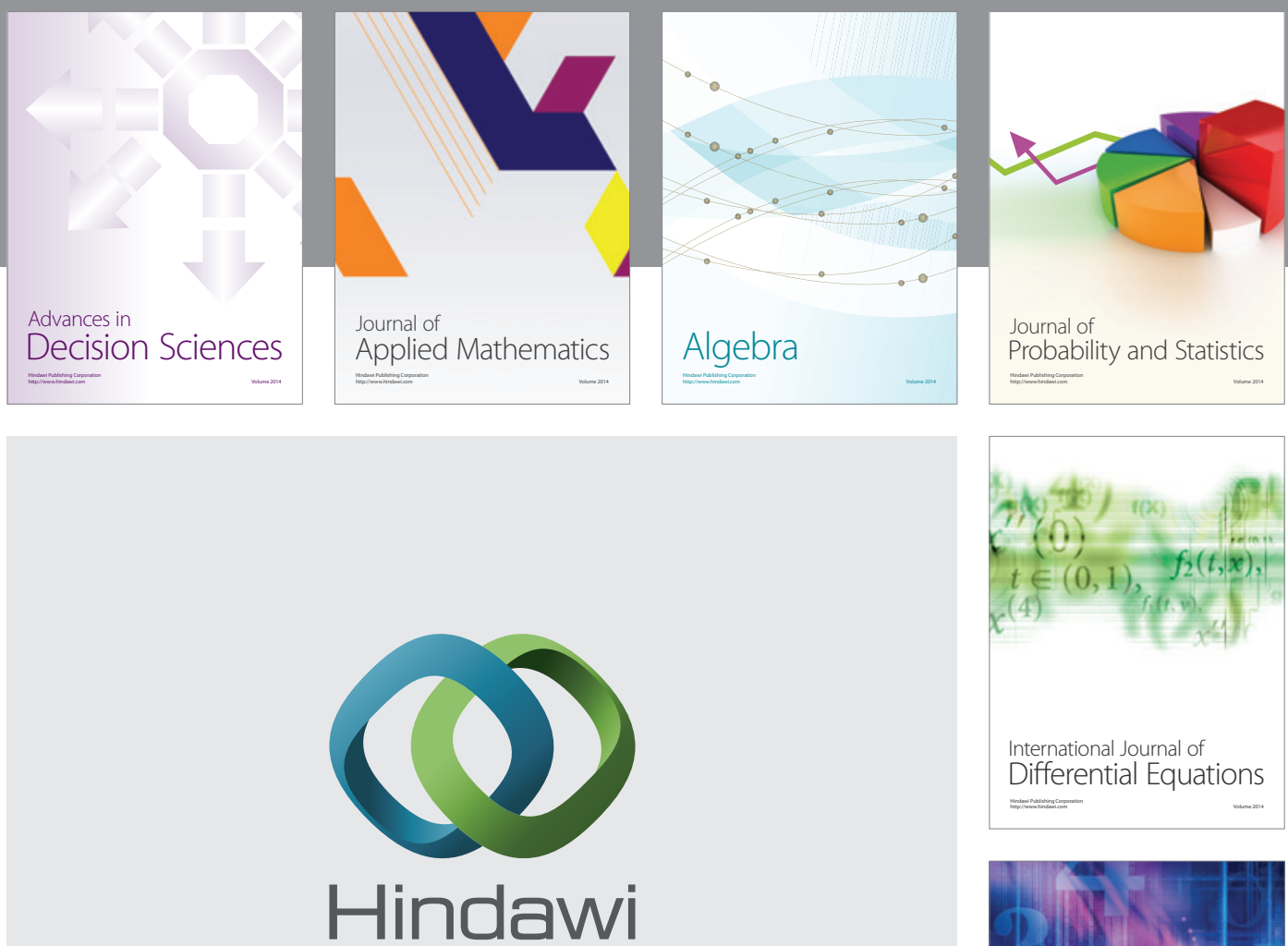

Submit your manuscripts at http://www.hindawi.com
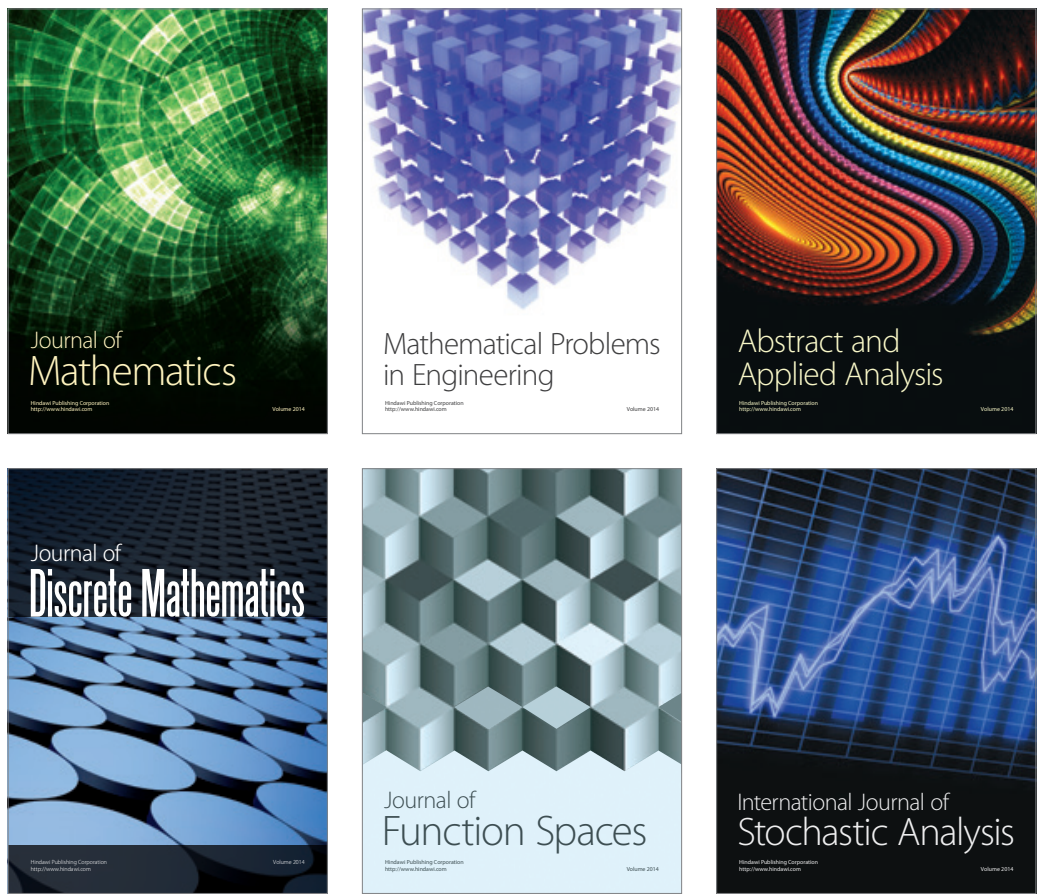

Journal of

Function Spaces

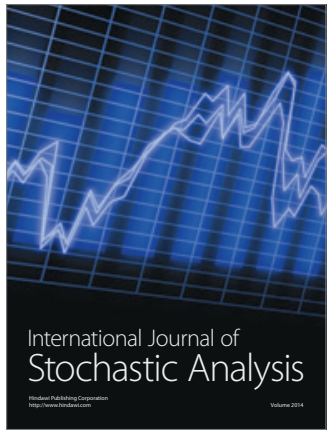

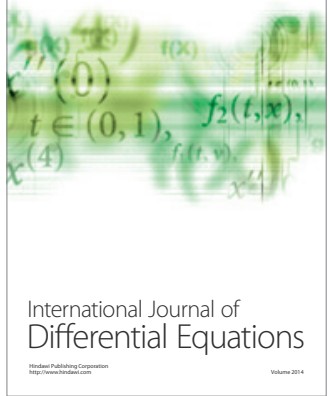
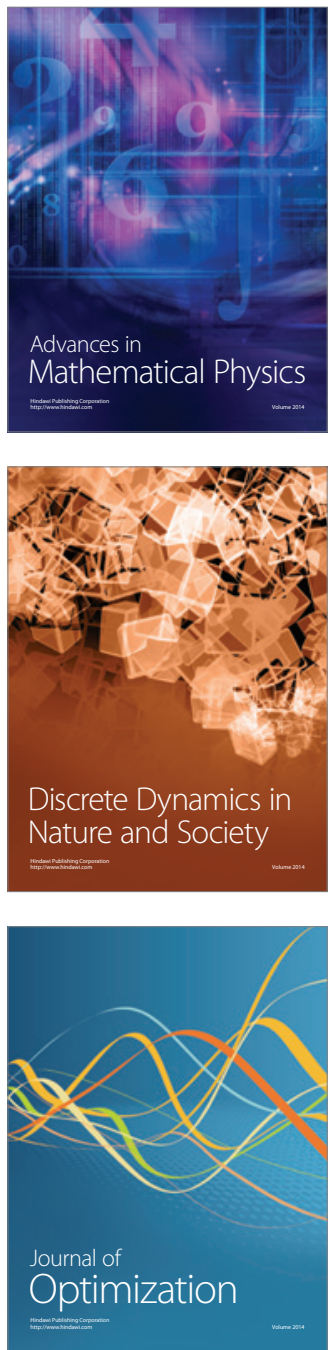